\title{
RESEARCH ON SEMANTIC MAP GENERATION AND LOCATION INTELLIGENT RECOGNITION METHOD FOR SCENIC SPOT SPACE PERCEPTION
}

\author{
Baishou Li ${ }^{1,2}$, Bing Liu ${ }^{1}$, Xiaoshan $\mathrm{Ni}^{1}$, Peng Huang ${ }^{1}$, Linlin $\mathrm{Pu}^{1}$ \\ ${ }^{1}$ College of Geomatics and Geoinformation, Guilin University of Technology, Guilin, Guangxi, China - (lbszhb, nbling288, \\ amapsx, hp04161018, 18376578436)@163.com \\ ${ }^{2}$ Guangxi Key Laboratory of Spatial Information and Geomatics, Guilin, Guangxi, China - lbszhb@163.comv
}

KEY WORDS: Semantic Map, feature extraction, Feature matching, Location perception

\begin{abstract}
:
In recent years, Tourism has become more and more Chinese leisure travel choice The research on the smart scenic spot is getting deeper and deeper, but the problem of accurate location 1 in the natural scenic spot still needs to be solved. Semantic maps contain a wealth of environmental information and can be more efficient for location-aware services, and are attracting more and more attention from researchers at home and abroad.

In order to better ensure the travel experience of tourists, the range of scenic spots is too large, and the signal interference is high. Complex terrain in the scenic area, Branch and leaf features Visitors cannot rely on traditional positioning systems to get their current accurate location. It is proposed to construct a navigation semantic map for the perception of scenic space. In the construction process, the operation based on the location perception of the tourists and the surrounding environment and the extraction of the feature information is the key to constructing the semantic map. The general image recognition method is used to obtain the environment image information, and the acquired feature image is recognized to obtain the semantic information in the environment; in order to obtain more feature environment information to better complete the location-aware service task, the GBP descriptor is used. The method divides and stores different semantic regions in the environment, and generates a semantic map with rich semantic information and feature information according to the three-dimensional map model.
\end{abstract}

\section{INTRODUCTION}

With the recent rapid development of location services and intelligent service technologies, and the increasing demand for positioning accuracy. Although traditional positioning technologies such as GPS have developed more maturely, they can meet most of the needs of people's daily life travel, but they still do not meet the needs of people's positioning and navigation in scenic tourism. Semantic map generation and location intelligence for scenic space perception The identification method, through the feature recognition, constructing the semantic map and the location recognition based on the intersection of the front, constructs the semantic map of the scenic spot, and provides the user with accurate location service, which satisfies the precise location information needs of people in the complex environment and situation of the scenic spot., enriched the smart phone positioning method.

Location-awareness is a location-based information service, which is a service that obtains location information through a mobile network, using a mobile device, and utilizing the positioning function of the mobile device itself. Spatial location database technology and communication service technology can be effectively integrated. Position sensing was originally used to improve the accuracy of industrial robots. Nowadays, with the continuous development of communication technology, mobile communication based on wireless networks has gradually replaced the communication mode based on fixed networks. At the same time, the widespread use of software such as GIS, GPSRS and VR using spatial information technology has enabled the rapid development of location-based services. Location awareness begins to shine in spatial positioning. In 2012, Wang Jian et al. proposed a vehicle-mounted mobile intelligent sensing and location service terminal device. ${ }^{[1]}$ The device includes various communication, positioning, interface and other modules. The device is easy to operate and can solve the needs of intelligent sensing and location services in the field of logistics and transportation. In 2013, it was proposed to collect human body position data through ultrasonic probes and transmit the data to the integrated chip controller. The integrated chip controller can calculate the real-time data of the actual position of the human body. ${ }^{[2]}$ The method aims to overcome the inconvenience of the conventional shower and to sense the human body. In 2015, an accurate position sensing system for indoor intelligent robots was proposed. ${ }^{[3]}$ This sensing system can sense its own attitude, including distance and azimuth, over a relatively long distance. Nowadays, it can be positioned by a single Beidou positioning module or positioning module, or any combination of three positioning modules can be used to achieve higher positioning accuracy. In recent years, ultra-wideband (UWB) high-precision real-time positioning technology has emerged. ${ }^{[4]}$ It is a carrierless, efficient, and highly reliable positioning technology. UWB (Ultra Wide Band) positioning technology is a carrierless communication technology that uses narrow pulses from nanoseconds to microseconds to transmit data. UWB is used for communication between devices, and the sub-nanosecond pulse signal can be used to measure the distance to achieve centimeter-level accuracy. Therefore, UWB communication is used for spatial positioning, and centimeter-level positioning accuracy can be obtained. The UWB pulse is rich in high-frequency components and can be directly radiated by the antenna. It does not require an RF carrier, and is most suitable for applications where security and confidentiality are required. The ultra-wideband location sensing system is divided into three parts: location information sensing layer, network transport layer and application layer. 


\section{GENERATION OF LOCATION-AWARE SEMANTIC}

\section{MAPS}

\subsection{Concept of Semantic Map of Scenic Spots}

Semantic maps are a regional location occupied by a versatile form of a specific language in a specific concept space, or a different way of cutting a different concept space in a certain language. ${ }^{[5]}$ 。 It consists of multiple types of information, which can link semantic information with database information to achieve efficient retrieval of content. It is a functional model presented by means of maps. It can be applied to many fields such as unmanned driving and VR technology.

Scenic Spot semantic map (Scenic Spot Semantic Maps) consists of time and space and semantic information of scenic spots. It can link the semantic information of scenic spots with the information of scenic spots database to realize efficient retrieval of scenic spot and space-time attribute content. It is a functional model expressed by means of maps.

\section{2 scenic area semantic map generation method}

1. Use the camera to capture the scenic image: use the camera to capture the basic semantic information of the image, the camera will first identify all the features of the same angle in the same area for storage. After that, the same storage method is performed from another angle. In the past, the semantic information of the entire area can be collected.

2. Observing point depth extraction feature: After the height matching and mass generation of feature information, other cameras will perform second imaging and scanning on the ground object, and obtain texture information, which will get multiple scans through different directions. The entire texture information of the object is extracted and stored. Building a Web program is based on GIS Sever. This program provides a comprehensive platform of location service, which includes location service system and information management system.

3. Classification and storage of data: According to different textures of different kinds of features, the image features can be specifically classified by using the GBP description sub-classification method, and stored in a database, which can be used to describe sub-categories based on the use of GBP description. The method is used to process the image texture features. The semantic information collected at this time conforming to the texture feature will be classified into the feature and the feature database is established.

Image classification is specifically classified by the GBP description sub-category method and stored in the database. ${ }^{[6]}$ 。 The semantic information collected at this time that conforms to this texture feature will be classified into the feature and a feature database will be established.

Zhou Li has proposed a classification method for GBP descriptors. ${ }^{[6]}$ The method is improved by the inspiration of the LBP descriptor. The Local Binary Pattern (LBP) is an operator used to describe the local texture features of the image.

The original LBP operator is defined for extractionThe middle square in the 9-pixel square is thresholded and will be adjacent.The gray value of 8 pixels is compared with the central pixel value, and the mark larger than the center point is 1 and less than 0 . After binary processing, the LBP value of the pixel center pixel of the pixel square can be obtained, and the value can be used. Reflects the texture information at the real scene. Expressed by the formula is:

$$
\operatorname{LBP}\left(\mathrm{X}_{\mathrm{C}}, \mathrm{Y}_{\mathrm{C}}\right)=\sum_{\mathrm{P}=0}^{\mathrm{P}-1} 2^{\mathrm{P}} \mathrm{S}\left(\mathrm{i}_{\mathrm{p}}-\mathrm{i}_{\mathrm{c}}\right)
$$

among them $\left(X_{c}, Y_{c}\right)$ is the center pixel, $i_{c}$ is the gray value, $i_{n}$ is the gray value of the adjacent pixel, $s$ is a symbol function

$$
\mathrm{s}(\mathrm{x})=\left\{\begin{array}{rr}
1 & \text { if } \mathrm{x} \geq 0 \\
0 & \text { else }
\end{array}\right.
$$

However, the LBP algorithm only compares the gray value between the pixels, but for the scenic area, the open-air illumination has a great influence on the gray value of the ground object extraction, and the texture feature information cannot be accurately distinguished. The robustness is strong. The child can improve this very well.

The GBP descriptor can be used to calculate the GBP value of all pixel points in a certain area of the partial image, regardless of the illumination factor, and is suitable for feature extraction and extraction of different time periods of the scenic spot map, and the extracted pixel value is represented by a histogram, The histogram vector can be used to describe the texture features of real objects in the scenic area. Normalize the computed histogram vector to get the final GBP descriptor.

Finally, the pixel value of each point is replaced by the calculated GBP value, and different scenic space semantic classification partitions are obtained to distinguish different scenes in the scenic area.

4. Discriminating the obstacle area: Judging whether there is a temporarily parked vehicle or a temporarily erected building in the photographed place.

The discriminating method is: checking whether the obtained texture feature is a texture feature existing in the database, and if there is an unrecognizable texture feature, the feature is deleted, and the feature is scanned and recognized until the obtained information is pure Semantic maps that can be queried, matched, and categorized in a semantic database.

5. Semantic map and panoramic image position matching: Semantic navigation map generated by the collected semantic information and position information of the electronic map collected by the panoramic collector are accurately matched with the position node.

6. Regularly update the map: at set intervals (this time node is the time node with large change of feature semantic information in the scenic area such as season), use the camera in the scenic spot to collect the panoramic map, update the database, and update the updated data information with The semantic information obtained initially is matched and merged.

\section{3 scenic area semantic map generation system}

The composition of the scenic area semantic map generation system is shown in Figure 1 include: 


\section{Semantic Feature Extraction Subsystem}

The semantic feature extraction subsystem is used to extract the feature information of the real scene image of the scenic spot, which can realize the detection and update of the feature of the scenic spot. It can also detect and determine the feature information of the scenic spot through the deep learning method, and update it in time. The updated feature information is combined with the original information.

\section{Semantic Feature Classification Subsystem}

The semantic feature classification subsystem is used to classify the extracted semantic information according to different feature features. For example, some scenic spots have lakes, mountains and other features with different semantic information stored in the database, according to different types of features. With different gray values, the image features are classified by the GBP description sub-classification method and stored in the real-time semantic map database.

\section{Semantic Information Storage Subsystem}

The real-time semantic information storage subsystem classifies the information classified by the semantic features into categories according to the categories, so that it can be extracted in the future.

\section{Semantic Map Data Management Subsystem}

The real-time semantic map data management subsystem is used to provide a platform for analysis and decision-making for the whole system. Based on the GIS server server, a web application is built, which provides a location service integrated platform, which includes a real-location service system and semantic map information. Management system.

\section{Scenic Area Semantic Computer Collaborative Labeling Automation Subsystem}

When the user sends the scene feature image after the scenic spot classification to the system, the system will perform semantic annotation word recommendation and prompting on the newly proposed semantic map feature requirement, and reduce the workload of creating the real scene semantic map. At the same time, multiple users can use the system to semantically mark word sharing and perform semantic-based feature retrieval and viewing.

6. Location Intelligent Recognition Cloud Computing Management Subsystem

The system is used to complete the fast calculation of the intelligent sensing and location service transmission network, and to ensure the management and maintenance of the hardware infrastructure.

\section{Scenic Area Semantic Map Building Module Subsystem}

The system is mainly responsible for merging the ground classification information and semantic information sent by the mobile terminal, and inserting it into the existing scenic area semantic map.

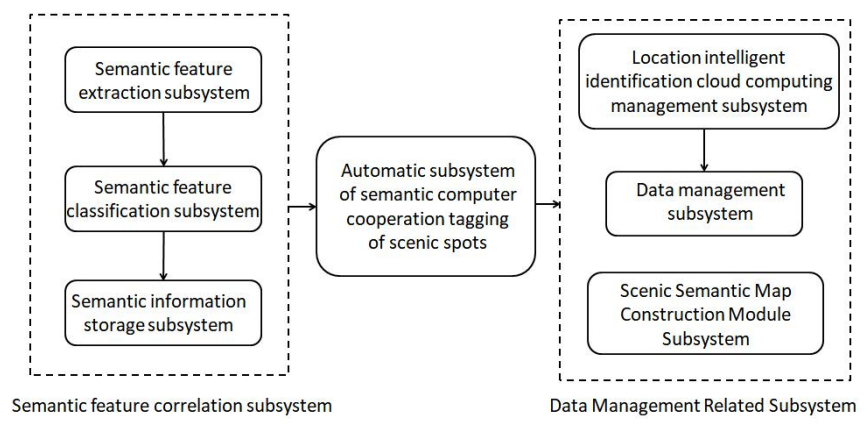

Figure 1 Scenic Spot Semantic Map Generation System

\section{LOCATION INTELLIGENT RECOGNITION METHOD BASED ON SCENIC AREA SEMANTIC MAP}

\subsection{Location intelligent identification method}

The method flow proposed by this paper includes: Firstly, the user targets a object in the certain location of it. By submitting a request for positioning to the system, two photos with a certain degreed of overlap taken by camera are sent to the system. Secondly, the system get a rough location where the user is, and establish a buffer with the location as the center, And then, texture extraction on one of the photos is performed and compared with the data of the feature database of the buffer range to find the location of corresponding building by the system. Finally, the system constructs a stereo pair through two photos, and calculates the precise position of the user in combination with the location of the object and the information of the stereo pair.

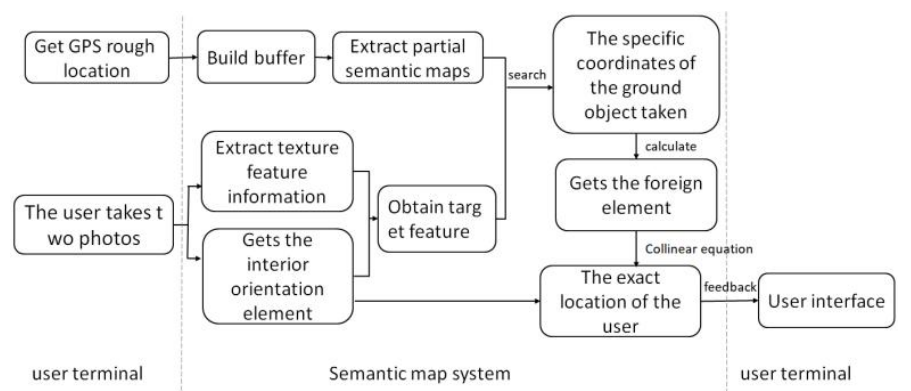

Figure 2 The Flowchart of The Intelligent Identification of Location

\subsection{Mobile-based scene matching and perceptual positioning}

1.Be Inaccurately positioned by GPS : The user's handheld terminal uses the terminal's GPS to obtain an inaccurate current coordinate. The network transmits sent the coordinates to the GIS spatial analysis system associated with the GIS Sever, and performs proximity analysis.: Centering on this coordinate, a buffer with a radius of $10 \mathrm{~m}$ is created, which is used as the range of the locality semantic map.

2. Extract the locality semantic map: The system connects the GIS spatial database containing the scenic map semantic map associated with the GIS Sever server, and uses the GIS spatial analysis system to retrieve all the features contained in the buffer range according to the coordinates, and extract the semantic map of these features to generate a locality semantic map. 
3. Collect feature of a object and match the information: the user uses the camera of the mobile phone to shoot two photos with a certain degree of overlap to form a stereo pair and stores it in the mobile phone. And then, by establishing a connection between the program and the mobile phone memory through the interface to create the application acquires. The information of the image, such as the pixel and the focal length $f$, and pass the information to the image processing system, using the image center point as the main point of image, that is, the coordinate origin $\mathrm{o}$, constructing the $\mathrm{x}$-axis, and the $\mathrm{y}$-axis direction is the same as the frame coordinate system. The Cartesian coordinate system o-xy indicates that the image point of the object is at the image plane. The coordinates of the image obtained by the first image are $(\mathrm{x} 1, \mathrm{y} 1)$, and the coordinates of the image of the second image are (x2, y2). In the feature extraction system, the texture descriptor is extracted by using the GBP descriptor for one of the photos, and the corresponding relationship between the texture feature and the coordinates is saved, and the corresponding GBP descriptor is found according to the coordinates of the principal point, in the local semantic map. Search for matching by setting a certain allowable value, find the GBP descriptor that meets the condition and find the center coordinate of the corresponding feature in the object space coordinate system (XA, YA, ZA)to determine the specific location of the object.

4. Using the stereo image pair to solve the user's specific location: Through the image processing system associated with the GIS Sever in the program, the stereo image pair constructed in step (3) is combined with the mobile phone sensor to perform the attitude angle element solution. to obtain the values of $\psi, \theta, \gamma .{ }^{[7]}$

The angle elements $\psi, \theta, \gamma$ obtained by the calculation can calculate the direction cosine values ai, bi, ci. Combine the image plane coordinates $\mathrm{x} 1, \mathrm{y} 1, \mathrm{x} 2, \mathrm{y} 2$ of the image point, focal length $f$, the object space coordinate XA,YA,ZA of the object obtained in step (3). The system calculate the specific location (X, Y, Z) of the user by using the collinear equation (2.1) and the calculation formula of the space front intersection principle.

$$
\left\{\begin{array}{l}
x_{1}=-f \frac{a_{1}\left(X_{A}-X\right)+b_{1}\left(Y_{A}-Y\right)+c_{1}\left(Z_{A}-Z\right)}{a_{3}\left(X_{A}-X\right)+b_{3}\left(Y_{A}-Y\right)+c_{3}\left(Z_{A}-Z\right)} \\
y_{1}=-f \frac{a_{2}\left(X_{A}-X\right)+b_{2}\left(Y_{A}-Y\right)+c_{2}\left(Z_{A}-Z\right)}{a_{3}\left(X_{A}-X\right)+b_{3}\left(Y_{A}-Y\right)+c_{3}\left(Z_{A}-Z\right)} \\
x_{2}=-f \frac{a_{1}\left(X_{A}-X\right)+b_{1}\left(Y_{A}-Y\right)+c_{1}\left(Z_{A}-Z\right)}{a_{3}\left(X_{A}-X\right)+b_{3}\left(Y_{A}-Y\right)+c_{3}\left(Z_{A}-Z\right)} \\
y_{2}=-f \frac{a_{2}\left(X_{A}-X\right)+b_{2}\left(Y_{A}-Y\right)+c_{2}\left(Z_{A}-Z\right)}{a_{3}\left(X_{A}-X\right)+b_{3}\left(Y_{A}-Y\right)+c_{3}\left(Z_{A}-Z\right)}
\end{array}\right.
$$

\subsection{Scenic Area Semantic Classification and Labeling}

The scene understanding of the scenic spot is to assign one or more semantic classification identifiers to the image, that is, to automatically mark the collected images according to a set of semantic classification identifiers given by the system. For example, coastlines, vehicles, houses, mountains, roads, etc.

There are two kinds of object recognition algorithms used in the construction of semantic maps: based on global features and local feature-based algorithms. However, using global-based algorithms, it is easy to be limited by the surrounding environment when collecting object features, and there is a possibility of data loss in complex scene recognition segmentation. The algorithm based on local features only needs to extract the obvious key feature points, and the effect displayed in two dimensions is better than the former.
Therefore, in the comparison between the two parties, the algorithm based on local features is selected.

The scenic scene based on the semantic map of the local feature refers to the classification of the extracted feature information according to the different types of image information of rivers, mountains, lakes, trees, etc. in the scene according to the language form. After the classification of semantic information, the local key features of the semantic map are marked.

Mojsilovic et al. proposed a method. ${ }^{[8]}$ First, the image is segmented according to the color and texture information to find the corresponding semantic identifier, and then the semantic classification information of the image is determined by using the semantic target. Through the above steps, the scenic area semantic map classification system and the user service system are constructed.

After obtaining the specific location information of the user, the system establishes a connection between the user mobile terminal and the GIS Sever server through the network, and extracts a map centered on the current specific location of the user and takes the screen size of the mobile phone as the center, and adopts a display processing system. The map view is fed back to the user via the network, and the user can perform other operations on the operation interface.

\section{CONCLUSION}

This paper proposes a method of constructing a semantic map by feature recognition and constructing a stereo pair to solve the user's specific location in the scenic spot. This method has several advantages over traditional positioning methods:

(1) The traditional positioning technology locates the handheld mobile terminal through GPS and other systems. Although the accuracy has been improved, it is prone to large errors in the complex environment and situation of the scenic spot. The positioning method defined in this paper mainly constructs the semantic map through feature recognition technology, and uses the semantic map to assist the positioning, which improves the positioning accuracy.

(2) Unlike the conventional stereo pair positioning technique, the conventional technique determines the coordinates of the target object by acquiring coordinates of the image point, coordinates of the movable device, camera focal length, and angular elements. The position-aware technique mentioned in this paper reverses the principle and inversely solves the coordinates of the device by acquiring the coordinates of the image point, the camera focal length and the corner element, and the coordinates of the target feature acquired by the constructed semantic map.

The main problems are as follows:

(1) When discriminating an obstacle area, objects with very similar semantic features may appear, resulting in a decrease in the accuracy of the judgment.

(2) In the position recognition process, since the coordinates of the center of the feature are used as the solution data, if the target object identified by the system is too large, the accuracy of the positioning may not be high. 


\section{ACKNOWLEDGMENTS}

This work was supported by the Guilin Scientific Research and Technology Development Plan(20190601; 2016012601), Guilin Guilin University of Technology Ph.D Scientific research initial funding(002401003316);Chongqing basic science and advanced technology research (cstc2015jcyjBX0023).Special thanks to the College of Geomatics and Geoinformation, Guilin University of Technology for the support of our work.

\section{REFERENCES}

[1] Wang Jian,Wang Haitao,Zou Guangnan. A Vehicle Mobile IntelliSense and Location Service Terminal Device: China, 201220250742.6[P].2012-12-26

[2] Luo Fuhui. A smart shower device that senses the position of the human body in real time: China, 201320582826.4[P].2014-03-12

[3] Wang Zhenxing. An accurate location sensing system for indoor intelligent robots and its sensing method: China, 201510206917.1[P].2015-12-16

[4] Ji Sujuan. A new method for positioning and navigation of intelligent agricultural robots [J].Modern trade industry,2016,37(2):226-227

[5] Shen Zhangrong. Questioning the Theory of Semantic Map Model[J]. Journal of Zhejiang Ocean University(Humanities Science Edition), 2013, 30(6): 61-67

[6] Zhou Li. Research on key technologies of natural scene classification and target recognition [D]. Harbin: National University of Defense Technology, 2012

[7] Li Zhaowei. Design and implementation of spatial front rendezvous positioning method based on Android mobile phone [D]. Jilin: Jilin University, 2017

[8] A. Mojsilovic, J. Gomes and B. Rogowitz. Isee: Perceptual features for image library navigation [C]. In Proceedings of SPIE Human vision and electronic imaging, San Jose, California, 2002:266-277. 